\title{
Analysis of a Proportionally Fair and Locally Adaptive Spatial Aloha in Poisson Networks
}

\author{
François Baccelli \\ University of Texas, Austin, USA \\ and Inria-ENS, France \\ Email: francois.baccelli@austin.utexas.edu
}

\author{
Bartłomiej Błaszczyszyn \\ Inria-ENS, France \\ 23 avenue d'Italie 75214 Paris \\ Email: Bartek.Blaszczyszyn@ens.fr
}

\author{
Chandramani Singh \\ Coordinated Science Lab \\ UIUC, Urbana, IL 61801, USA \\ Email: chandramani.singh@inria.fr
}

\begin{abstract}
The proportionally fair sharing of the capacity of a Poisson network using Spatial-Aloha leads to closed-form performance expressions in two extreme cases: (1) the case without topology information, where the analysis boils down to a parametric optimization problem leveraging stochastic geometry; (2) the case with full network topology information, which was recently solved using shot-noise techniques. We show that there exists a continuum of adaptive controls between these two extremes, based on local stopping sets, which can also be analyzed in closed form. We also show that these control schemes are implementable, in contrast to the full information case which is not. As local information increases, the performance levels of these schemes are shown to get arbitrarily close to those of the full information scheme. The analytical results are combined with discrete event simulation to provide a detailed evaluation of the performance of this class of medium access controls.
\end{abstract}

\section{INTRODUCTION}

This work is focused on a version of Spatial Aloha [4] where each node willingly controls his medium access probability (MAP) in order to maximize the network-wide sum of the logarithms of the node throughputs, a global objective referred to as proportional fairness in the literature. A good analogy is that of TCP users who willingly throttle their transmission rates so as to maximize some network wide utility [13]. In TCP, the transmission control is both adaptive and decentralized: it is decentralized since each user throttles his rate based on his own packet losses, which are seen as an indicator of the presence of contending users; it is adaptive in that the throttling is substantial if there are many contenders (and hence losses) at any given time and small otherwise. In the version of Spatial Aloha studied in this paper, the access control is also decentralized and adaptive: it is decentralized because each node computes its MAP using an equation based on some local spatial information subsuming its wireless contention status; it is adaptive w.r.t. local spatial conditions in that a node will apply an heavy throttling if there are many wireless receivers nearby, and a small one (or none) otherwise.

Stochastic geometry [3] has recently been used for the analysis and performance evaluation of wireless (ad hoc as well as cellular) networks; in this approach, one models node locations as a spatial point process, e.g., a homogeneous Poisson point process (PPP), and one computes various network statistics, e.g., the distribution of interference, the successful transmission probability, the coverage (or outage) probability etc. as spatial averages. This leads to closed form expressions for a variety of performance metrics that are then amenable to optimization with respect to network parameters (node density, protocol parameters, etc.) [2]. In the Aloha case, this approach takes a macroscopic view of the network with the underlying assumption, justified by homogeneity, that all nodes in the network have identical statistical characteristics. The parameter to be optimized is the MAP, and at the optimum operation point, all users have the same MAP as a direct consequence of homogeneity.

Analyzing the behavior of the adaptive version of Spatial Aloha described above in the context of large random networks requires extending stochastic geometry to the situation where each node selects a MAP resulting from a global optimization. This is not an easy task in general as optimization schemes aiming at maximizing some global utility lead to intricate correlations between the behaviors of all nodes and these correlations most often prevent the use of the independence based tools underlying most of random graph theory and stochastic geometry.

The present paper identifies a class of local adaptation policies for which the optimal node MAP control leads to closed form expressions for both the behavior of a typical node and global performance metrics; these closed forms are obtained under the assumption of an infinite random network whose nodes have locations forming a realization of a homogeneous PPP in the Euclidean plane. These adaptive policies are defined in term of stopping sets (see below). A typical example of stopping set is the smallest disk that contains the $k$ closest receivers/neighbors of a node. The associated adaptive policy consists in having each transmitter controlling his MAP as a function of the actual locations of the $k$ closest receivers which are the closest to its own location and the estimated density of the other receivers, and in adopting the MAP which maximizes the sum of the logarithms of the rates of all nodes in view of this information and estimation. Another example of stopping set is any deterministic set (e.g. a disk). The class is however much larger than what is suggested by these two simple examples, as we shall see below.

So far only two extreme cases are understood within this general adaptive Aloha framework: (1) the case with empty stopping sets: this form of open loop control can be analyzed using the tools of stochastic geometry and parametric optimization alluded to above; (2) the case with $\mathbb{R}^{2}$ stopping sets: this form of adaptive control, which is based on full infor- 
mation on the network topology, was quite recently analyzed in [6]. As in many optimal control problems, none of these extreme cases are really satisfactory. The former is suboptimal: it is well known that the knowledge of actual location information should allow one to quite significantly improve overall network performance; the latter is not implementable as it requires location informations of all the nodes in the network (each transmitter has to acquire knowledge on either the locations of all receivers or its path-loss to all receivers, which form infinite sequences in both cases).

The main new mathematical results obtained in the present paper are (i) the characterization of the optimal policy for any (translation invariant) stopping set information structure [Theorem 3.1] and (ii) closed form expressions for the resulting performance metrics [Theorems 4.1-4.4 below]. These, in the first place, constitute an important theoretical extension of what is currently known on Spatial Aloha. In addition, as we shall see, the locality of the stopping set can be used to define implementable control schemes, and our analytical tools can also be used to find a good trade-off between the quality of the optimal strategy and the locality of the stopping sets.

Let us now give a somewhat broader review of the state of the art on the performance of Aloha, Spatial Aloha and related protocols. Aloha and slotted Aloha were introduced and analyzed by Abramson [1] and Roberts [15] respectively. In these protocols, only one node could successfully transmit at a time. In Spatial Aloha, as considered in [4], nodes are located in the Euclidean space, signal power is attenuated according to some path-loss function and the success of each transmission is based on Signal to Interference and Noise Ratio (SINR). This leads to an essential property of wireless networks which is spatial reuse, namely the fact that infinitely many simultaneous successful transmissions can take place in the whole space, provided they are "well separated". More precisely, it was shown that such networks can sustain a positive spatial density of successful transmissions [4]. All the above protocols prescribe identical MAPs for all nodes.

Among the initial attempts of MAP adaptation in Aloha, let us first quote [9] which analyzes stochastic approximation based strategies adapting the MAP to receiver feedbacks and aiming at stabilizing the network. This approach was considered in the spatial random network context in [7] for the protocol model but not for the SINR setting. The other recent publications on the matter can be organized as follows: (1) Adaptation to local channel conditions. In spatial random networks with SINR based reception, such local adaptations are amenable to parametric optimization as described above [5, 12]. Some versions of this type of adaptation are referred to as Oppotunistic Aloha [2]. The case with no dependence on geometry (i.e., no path-loss component) is also considered in [11] where a paradoxical behavior is identified for certain topologies: plain Aloha yields better aggregate throughput than opportunistic Aloha. (2) Centralized optimization for fixed topologies. The case with SINR based reception is studied in [14], where the authors give centralized algorithms determining the random access probabilities that maximize either the total network throughput or lead to a max-min fair operation. By nature, this approach is difficult to use in large random spatial networks. (3) Interference graph optimization. There is a vast literature on this topic which replaces SINR reception by exclusion rules on a graph. Among the most relevant papers for our purposes, let us quote [16] which proposes algorithms that lead to either proportional fairness or max-min fairness and [8] which derives the Pareto boundary of the rates achievable by Aloha. (4) Game theoretic analysis. In this appraoch, the MAP adaptation is modeled by a game. Again, we will limit ourselves to papers investigating the case of spatial random networks. The most relevant references are [10] and [17]. Reference [10] formulates the channel access problem as a non-cooperative game among users and proposes pricing schemes that induce a socially optimum behavior at equilibrium. However the approach is restricted to symmetric Nash equilibria, which forbids the type of non-symmetrical adaptations of interest here. Reference [17] shows that Aloha is often a Pareto optimal strategy when users aim at selecting their transmission powers in a game theoretic way. A key difference between game theory and the line of thought of the present paper is that users are selfish in the former and altruistic in the latter (as explained above, as in TCP, they willingly throttle their rate to maximize some utility).

Let us summarize this survey by stressing that, excluding [6] discussed above, none of the above references considers a large random Aloha network where nodes adapt to wireless channel randomness and to spatial fluctuations of topology for making non asymmetric random access decisions maximizing some utility, as we do in the present paper.

There is also a vast literature on the modeling of CSMA in large random networks by stochastic geometry. In spite of the fact that the very nature of this MAC protocol is adaptive, we will not discuss this here since CSMA is only designed to guarantee a reasonable scheduling, not to optimize any utility.

The present paper is structured as follows: the network setting is described in Section II. There, we describe the quasi-static networks of interest, in which mobiles learn the local topology and incorporate this information in their MAP selection. Section III is focused on the stopping set based distributed algorithms that lead to a proportional fair sharing of the network resources. We show that nodes can compute the optimal MAPs as solutions to certain fixed point equations. Section IV contains the analytical performance results. For nodes forming a realization of a homogeneous Poisson Point Process (PPP) in the Euclidean plane, we compute the MAP distribution using the theory of shot noise fields. Using this distribution, we also derive the mean utility of a typical node. The numerical results are gathered in Section V. The aim of this section is three-fold: (1) validate the analytical results against simulation; (2) quantify the gains brought by adaptation; (3) determine a good trade-off between information and performance. Finally, we discuss the implementability of this class of controls in Section VI. 


\section{NETWORK MOdEL}

We model the ad-hoc wireless network as a set of transmitters and their corresponding receivers, all located in the Euclidean plane. This is often referred to as the "bipole model" [2, Chapter 16]. We assume that each node has an infinite backlog of packets to transmit to its receiver. The transmitters follow the slotted version of the Aloha medium access control (MAC) protocol. A transmitter, in each transmission attempt, sends one packet which occupies one slot. The transmission succeeds if the signal to noise plus interference ratio (SINR) at the corresponding receiver exceeds a designated threshold.

We assume that the transmitting nodes are scattered according to a homogeneous Poisson point process of intensity $\lambda$. Each transmitter is associated with a multidimensional mark that carries information about the location of the corresponding receiver, the fading conditions of the channels to all the receivers and about its MAC status at the current time slot. More precisely, we have at our disposal a marked Poisson point process $\tilde{\Phi}=\left\{X_{i}, y_{i}, \mathbf{F}_{i}, e_{i}, p_{i}\right\}$. Below,

- $\Phi=\left\{X_{i}\right\}$ denotes the PPP of intensity $\lambda$ representing the location of transmitters in $\mathbb{R}^{2}$.

- $\bar{\Phi}=\left\{y_{i}\right\}$ denotes the locations of the receivers. The receiver of transmitter $X_{i}$ is $y_{i}$. For notational convenience, we also define random vectors $\left\{r_{i}=y_{i}-X_{i}\right\}$. We assume that $\left|r_{i}\right|=r$, a constant, and that $\left\{\angle r_{i}\right\}$ are i.i.d. and uniform on $[0,2 \pi]$ and independent of $\Phi$. From the displacement theorem [3], $\bar{\Phi}$ is also a homogeneous PPP of intensity $\lambda$.

- $\left\{\mathbf{F}_{i}=\left(F_{i}^{j}: j\right)\right\}$ where $F_{i}^{j}$ denotes the random fading between transmitter $j$ and receiver $i$. We assume that channels are Rayleigh faded and that the random variables $\left(F_{i}^{j}: i, j\right)$ are independent and exponentially distributed with mean $1 / \mu$. The independence of the components of $\left(F_{i}^{j}: i, j\right)$ is justified in moderately dense networks.

- $\left\{e_{i}\right\}$ are indicators that take value one if the corresponding node decides to transmit in the considered time slot, and zero otherwise.

- $p_{i}$ denotes the MAP of node $i: p_{i}=\mathbb{P}\left(e_{i}=1\right)=1-$ $\mathbb{P}\left(e_{i}=0\right)$. Also, given $p_{i}, e_{i}$ is independent of everything else including $\left\{e_{j}\right\}_{j \neq i}$.

We assume that each transmitter uses unit transmission power. We also adopt an omnidirectional path-loss model with power attenuation given by $l(z)=z^{-\beta}$ for a $\beta>2$. The receivers are also subjected to white Gaussian thermal noise with variance $W$, which is also constant across slots. Thus, when a transmitter $i$ transmits, the SINR at its receiver is

$$
\operatorname{SINR}_{i}=\frac{F_{i}^{i} r^{-\beta}}{\sum_{j \neq i}\left|X_{j}-y_{i}\right|^{-\beta} F_{j}^{i} e_{j}+W},
$$

where the first term in the denominator is the power of the interference at receiver $i$, which is a shot noise associated with $\tilde{\Phi}$ (see e.g. [3]). We assume that a transmission from $i$ to its receiver is successful if $\operatorname{SINR}_{i}$ exceeds some threshold $T$.

\section{Proportionally Fair Aloha}

The principle of proportional fairness [13] consists in maximizing the sum of the logarithms of user throughputs.

\section{A. Node Throughput}

In our model, the throughput of transmitter $i$ (actually, transmitter-receiver pair $i$ ) is $p_{i} q_{i}$, where $q_{i}$ is the probability of successful transmission of node $i$ given this node is authorized to transmit. More specifically, we consider the conditional probability of successful transmission of node $i$ given the network geometry $\Phi, \bar{\Phi}: q_{i}=q_{i}(\Phi, \bar{\Phi}):=$ $\mathbb{P}\left\{\operatorname{SINR}_{i} \geq T \mid \Phi, \bar{\Phi}\right\}$. Let $b_{j i}:=\left|X_{j}-y_{i}\right|^{\beta} /\left(\operatorname{Tr}^{\beta}\right)$. The conditional probabilities $q_{i}$ admit the following expression, valid for arbitrary (fixed) network topology $\Phi, \Phi$, with either a finite or an infinite number of nodes:

\section{Lemma 3.1:}

$$
q_{i}=e^{-\mu \operatorname{Tr}^{\beta} W} \prod_{j \neq i}\left(1-\frac{p_{j}}{1+b_{j i}}\right) .
$$

Proof: Condition first on $\Phi, \bar{\Phi}$ and $\mathcal{F}_{i}=\left\{F_{j}^{i}, e_{j}, j \neq i\right\}$ :

$$
\begin{aligned}
& \mathbb{P}\left\{\operatorname{SINR}_{i} \geq T \mid \Phi, \bar{\Phi}, \mathcal{F}_{i}\right\} \\
& =\mathbb{P}\left\{F_{i}^{i} \geq T r^{\beta}\left(\sum_{n \neq i}\left|X_{j}-y_{i}\right|^{-\beta} F_{j}^{i} e_{j}+W\right) \mid \Phi, \bar{\Phi}, \mathcal{F}_{i}\right\} \\
& =e^{-\mu T r^{\beta} W} e^{-\sum_{j \neq i} \frac{\mu T r^{\beta} F_{j}^{i} e_{j}}{\left|X_{j}-y_{i}\right|^{\beta}}},
\end{aligned}
$$

where the last expression follows from the fact that $F_{i}^{i}$ is exponential, independent of $\mathcal{F}_{i}$. Averaging over $\mathcal{F}_{i}$, we obtain

$$
\prod_{j \neq i} \mathbb{E}\left[e^{-\frac{\mu T r^{\beta} F_{j}^{i} e_{j}}{\left|X_{j}-y_{i}\right| \beta}}\right]=\prod_{j \neq i}\left(1-p_{j}+\frac{p_{j}}{1+1 / b_{j i}}\right) .
$$

We obtain (1) by further simplifying the product factors.

Thus the proportional fairness of our model consists in finding the $p_{i} \mathrm{~s}, 0 \leq p_{i} \leq 1$, which maximize the sum of the logarithms of the throughputs $\sum_{i} \log \left(p_{i} q_{i}\right)$. However, in infinite networks, this sum is typically unbounded. In particular, it is unbounded for almost all realizations of our Poisson network of Section II. Below, we first propose a specific formulation of the proportional fairness in the context of an infinite ergodic model. Subsequently, we show that the solution of this problem asymptotically coincides with a meaningful maximization of the sum of the logarithms of the throughputs in a finite network when its size tends to infinity.

\section{B. MAC Policies}

Denote by $\mathcal{S}_{a}$ the shift operator on $\Phi, \bar{\Phi}$. It translates all the network nodes by the vector $-a: \mathcal{S}_{a}\left\{X_{i}\right\}=\left\{X_{i}-a\right\}$ and similarly for the receivers, preserving all the nodes characteristics. We extend this operator to all (random or deterministic) subsets $A \subset \mathbb{R}^{2}$ by defining $\mathcal{S}_{a} A=\{x-a: x \in A\}$. An important assumption in the infinite model is that all nodes behave in the same way if they thy see the same configuration of nodes in $\Phi, \bar{\Phi}$. More precisely, by Translation invariant MAC policy, we mean a policy where all nodes $X_{i}$ set their MAPs to $p_{i}=\psi\left(\mathcal{S}_{X_{i}} \Phi, \mathcal{S}_{X_{i}} \bar{\Phi}\right)$, where $\psi(\cdot)$ is some given 
function which takes as its argument the network geometry $(\Phi, \bar{\Phi})$ and has its values in $[0,1]$. In other words, any node $X_{i}$, in order to chose its MAP, applies the same MAC policy $\psi$ evaluated for the network geometry "seen" from its point of view, i.e. from $X_{i}$.

Usually a given node will only have some partial information about the location of other nodes, e.g. limited to some geometric vicinity. The notion of "local spatial information" can be formalized using the notion of stopping set $S=S(\Phi, \bar{\Phi})$ (cf. [3, Definition 1.9]). This is a subset of the plane $\mathbb{R}^{2}$ such that for any observation window $A$ one can determine whether $S(\Phi, \bar{\Phi}) \subset A$ when knowing only the points of $\Phi, \bar{\Phi}$ in $A .{ }^{1}$ The stopping set $S$ models the region in which the locations of nodes are known to a (hypothetical) observer located at the origin. In this context, it is natural (but not necessary) to assume that $S$ is some neighborhood of the origin. The simplest examples of $S(\Phi, \bar{\Phi})$ are disks centered at the origin of fixed radius $R$ or of radius equal to the distance to the $n-$ th closest transmitter, or $n$-th closest receiver or $n$-th closest node (regardless whether it is a transmitter or a receiver).

For a given stopping set $S=S(\Phi, \bar{\Phi})$ we consider the following class of MAC policies with local spatial information $S$. By this we mean a (translation invariant) MAC policy $\psi$ satisfying the following constraint

$$
\psi(\Phi, \bar{\Phi})=\psi(\Phi \cap S, \bar{\Phi} \cap S) .
$$

Note that in order to apply such a policy and evaluate its MAP $p_{i}=\psi\left(\mathcal{S}_{X_{i}} \Phi, \mathcal{S}_{X_{i}} \bar{\Phi}\right)=\psi\left(\mathcal{S}_{X_{i}} \Phi \cap S_{i}, \mathcal{S}_{X_{i}} \bar{\Phi} \cap S_{i}\right)$, node $X_{i}$ needs to know only the locations of the other nodes in the stopping set $S_{i}:=S\left(\mathcal{S}_{X_{i}} \Phi, \mathcal{S}_{X_{i}} \bar{\Phi}\right)$ (e.g. in a fixed disk around it, the disk up to its $n$-th closest neighbor, etc.)

\section{Infinite Network Optimization under Spatial Constraints}

Denote by $\mathbb{P}^{0}$ the Palm distribution of the stationary marked point process $\tilde{\Phi}$. Recall that almost surely under $\mathbb{P}^{0}$ there is a node located at the origin $X_{0}=0$, called the typical node. By Slivnyak's theorem, in the case of the Poisson process, this is just an "extra" node added at the origin to the stationary configuration of nodes, with all its characteristics independent and distributed identically as for all other nodes. Denote by $\mathbb{E}^{0}$ the expectation with respect to $\mathbb{P}^{0}$.

Let a stopping set $S=S(\Phi, \bar{\Phi})$ be given. Denote by $\mathbf{P} \mathbf{F}^{S}$ the following maximization problem in the MAC policy $\psi(\cdot)$

$$
\begin{aligned}
& \mathbf{P F}^{\mathrm{S}} \text { : maximize } \mathbb{E}^{0}\left[\log \left(p_{0} q_{0}\right)\right] \\
& \text { subject to } \quad 0 \leq p_{i}=\psi\left(S_{X_{i}} \Phi, S_{X_{i}} \bar{\Phi}\right) \leq 1 \\
& \text { and } \psi(\cdot) \text { satisfying (2). }
\end{aligned}
$$

We call $\mathbf{P} \mathbf{F}^{S}$ the proportional fair Aloha problem with spatial information $S$.

Note that under $\mathbb{P}^{0}$ we have $p_{0}=\psi=\psi(\Phi, \bar{\Phi})$ and that (3) corresponds to the maximization of the expected logarithm of the throughput of the typical node. The fact that the maximization is done with respect to a policy $\psi(\cdot)$ applied

\footnotetext{
${ }^{1}$ The notion of stopping set (with respect to a spatial point pattern) is a spatial analogue of the stopping time for a temporal process.
}

by all the nodes in the network makes it non-trivial, despite the fact that we maximize the utility function for just one node $X_{0}=0$. Note also that $\mathbb{E}^{0}\left[\log \left(p_{0} q_{0}\right)\right] \leq 0$.

For a given stopping set $S=S(\Phi, \bar{\Phi})$ consider the following MAC policy. Define $\psi^{S}$ as the (unique) solution of

$$
\frac{1}{\psi}=\sum_{y_{j} \in S, j \neq 0} \frac{1}{1+b_{0 j}-\psi}+\int_{y \in \mathbb{R}^{2} \backslash S} \frac{\lambda \mathrm{d} y}{1+|y|^{\beta} / \operatorname{Tr}^{\beta}-\psi}
$$

in $\psi$ provided

$$
a_{i}:=\sum_{y_{j} \in S, j \neq 0} \frac{1}{b_{0 j}}+\int_{y \in \mathbb{R}^{2} \backslash S} \frac{\lambda \mathrm{d} y}{|y|^{\beta} / T r^{\beta}}>1
$$

and $\psi^{S}=1$ otherwise. The existence and uniqueness follow from the fact that the L.H.S. of (4) decreases from $\infty$ to 1 w.r.t. $\psi$ on $[0,1]$ whereas the right hand side (R.H.S.) increases to $a_{i}$ on $[0,1]$, and from the continuity of these two functions.

Note that $\psi^{S}$ satisfies (2). In fact $\psi^{S}(\Phi, \bar{\Phi})=\psi(\bar{\Phi} \cap S)$ depends only on the configuration of receivers in $S$.

We now state now the main structural result of this paper. Its proof is given in Appendix.

Theorem 3.1: For all given stopping sets $S=S(\Phi, \bar{\Phi})$, the MAC policy $\psi^{S}$ defined by the fixed point equation (4) is a solution of the proportional fair Aloha problem $\mathbf{P} \mathbf{F}^{S}$ with spatial information $S$. For this MAC policy $-\mathbb{E}^{0}\left[\psi^{S} q_{0}\right]<\infty$. Moreover, for any MAC policy $\psi^{\prime}(\cdot)$ solving $\mathbf{P} \mathbf{F}^{S}$ we have $\psi^{\prime}(\Phi, \bar{\Phi})=\psi^{S}(\Phi, \bar{\Phi})$ for almost all realizations of $(\Phi, \bar{\Phi})$.

\section{Extended Window Approach}

The proportional fair medium access problem is usually stated as follows in the context of a finite subset of network nodes. Consider some selected (arbitrarily chosen) nodes $X_{1}, \ldots, X_{N}$ from $\Phi$, with their MAPs $p_{i}$ and success probabilities $q_{i}$ given by (1). Consider the optimization problem

$$
\begin{aligned}
& \mathbf{P F}^{*}: \text { maximize } \sum_{i \in[N]} \log \left(p_{i} q_{i}\right) \text {, in }\left\{p_{i}\right\} \\
& \text { subject to } 0 \leq p_{i} \leq 1, \forall i \in[N] \text {, }
\end{aligned}
$$

where $[N]=\{1, \ldots, N\}$. Note that here, we do not restrict ourselves to translation invariant MAC policies. $\mathbf{P F}^{*}$ is a convex separable optimization problem. The optimal MAPs can be characterized as follows, cf. [6]: the unique solution of $\mathbf{P F}^{*}$ is the unique solution $p_{i}, 1 \leq i \leq N$, of

$$
\frac{1}{p_{i}}=\sum_{j \in[N] \backslash\{i\}} \frac{1}{1+b_{i j}-p_{i}},
$$

provided $a_{i}=\sum_{j \in[N] \backslash\{i\}} 1 / b_{i j}>1$ and $p_{i}=1$ otherwise.

The thermal noise $W$ appears merely in a constant additive term in the objective function. So, this does not affect the optimal MAPs. For the same reason interferers $X_{j}, j \notin[N]$, external to the selected subset of nodes $X_{1}, \ldots, X_{N}$, do not affect the optimal MAPs of these nodes. As in the previous section, the optimal MAP of a transmitter $i$ is only a function of other receivers' locations $\left\{y_{j}\right\}_{j \in[N] \backslash\{i\}}$. In particular, given $\left\{y_{j}\right\}_{j \in[N] \backslash\{i\}}, p_{i}$ does not depend on $\left\{X_{j}\right\}_{j \in[N] \backslash\{i\}}$.

Now, consider a bounded observation window $A$ on the plane and the solution $\left\{p_{i}^{A}: X_{i} \in A\right\}$ of the $\mathbf{P F}^{*}$ problem 
posed for the (finite set of) nodes $X_{i} \in A$. Call it $\mathbf{P F}{ }^{\mathrm{A}}$ problem. The following corollary follows immediately from of the above characterization of the solution of $\mathbf{P F}^{*}$ :

Corollary 3.1: For any given $X_{i} \in \Phi$, when $A$ increases to $\mathbb{R}^{2}, p_{i}^{A}$ converges to $p_{i}^{\mathbb{R}^{2}}$ which is the unique solution (in $p_{i}$ ) of (7) with the summation carried out over all $j \neq i$.

Remark 3.1: Note that $p_{i}^{\mathbb{R}^{2}}=\psi^{\mathbb{R}^{2}}\left(\mathcal{S}_{X_{i}} \Phi, \mathcal{S}_{X_{i}} \bar{\Phi}\right)$, i.e., the solution of the proportional fair Aloha problem with complete geometry information $\left(S=\mathbb{R}^{2}\right)$ considered in Section III-C prescribes MAPs that are equal to the limits of the solutions of the finite problem $\mathbf{P} \mathbf{F}^{\mathrm{A}}$ when $A \rightarrow \mathbb{R}^{2}$.

Finally, the maximization of the mean utility of the typical node (3) can be also interpreted (via ergodicity) as the spatial average of node utilities in the extended window. Indeed, note that the maximization of $\sum_{X_{i} \in A} \log \left(p_{i} q_{i}\right)$ for a given bounded window $A$ is equivalent to that of $\Theta_{A}=$ $1 /(\lambda|A|) \sum_{X_{i} \in A} \log \left(p_{i} q_{i}\right)$, where $|A|$ is the surface of $A$ and $\lambda$ is the transmitter density. When $A \rightarrow \mathbb{R}^{2}, \Theta_{A}$ converges to $\mathbb{E}^{0}\left[\log \left(p_{0} q_{0}\right)\right]$ in the case when $\psi_{i}=\psi\left(\mathcal{S}_{X_{i}} \Phi, \mathcal{S}_{X_{i}} \bar{\Phi}\right)$.

\section{E. Examples of Stopping Sets}

We illustrate this via a few examples. We limit ourselves to disk based sets, which makes sense in the isotropic and omnidirectional setting considered here. But the proposed framework is quite versatile and could accommodate more general situations. We start with deterministic stopping sets and then consider random ones. In both cases, we illustrate the continuum alluded to above by increasing levels of information.

Below, we will denote by $R_{p}$ the distance between $X_{0}$, the tagged transmitter, and its $p$-th closest receiver, excluding $y_{0}$, with $p$ a positive integer. Let $F(\psi, S)$ denote the R.H.S. of (4) for a general stopping set $S$. With this notation, the tagged transmitter's MAP satisfies the fixed point equation

$$
\frac{1}{\psi}=F(\psi, S)
$$

We will use the following notation:

$$
\begin{aligned}
& D(\psi, S)=\sum_{j \neq 0: y_{j} \in S} \frac{1}{\frac{\left(\left|y_{j}\right| / r\right)^{\beta}}{T}+1-\psi}, \\
& C(\psi, x)=2 \pi \lambda r^{2} \int_{x}^{\infty} \frac{s}{\frac{s^{\beta}}{T}+1-\psi} \mathrm{d} s .
\end{aligned}
$$

1) $S=\emptyset$ : This is the case where transmitters have no topological information at all. In this case, all transmitters use an identical MAP given by (8) with $F(\psi, S)=C(\psi, 0)$. Notice that in this case, $\psi<1$ irrespective of the node density $\lambda$. Further, this MAP is different from the one that maximizes the density of successful transmissions (see [2, Section 16.3.1.1]), which makes sense as the objective functions of the parametric optimization are different. For the special case $\beta=4$, we get:

$$
\psi^{\emptyset}=\frac{\sqrt{1+4 \alpha^{2}}-1}{2 \alpha^{2}}, \quad \text { where } \quad \alpha=\frac{\pi^{2} \lambda r^{2} \sqrt{T}}{2} .
$$

2) $S=B_{0}(R)$ : In this example, each transmitter knows the locations of all receivers in a disk of radius $R$ centered on its location, with $R$ a constant parameter in $(0, \infty)$. This parameter can be tuned to control the mean cardinality of the set of receivers taken into account in the adaptive control. An inconvenience of this setting is that this cardinality is random (it follows a Poisson law). The parameter $R$ can also be used in order to upper-bound by $R^{\beta}$ the mean path-loss between the tagged transmitter and this set of receivers. This will be important in the proposed implementation (see Section VI). In this case $\psi$ is solution of (8) with

$$
F(\psi, S)=D\left(\psi, B_{0}(R)\right)+C\left(\psi, \frac{R}{r}\right) .
$$

For $\beta=4$, we can further simplify the second term as

$$
C\left(\psi, \frac{R}{r}\right)=\frac{\pi \lambda r^{2} \sqrt{T}}{\sqrt{1-\psi}}\left(\frac{\pi}{2}-\tan ^{-1}\left(\frac{(R / r)^{2}}{\sqrt{T(1-\psi)}}\right)\right) .
$$

3) $S=\mathbb{R}^{2}$ : Here, each transmitter knows and uses the location information of all receivers in the network. In this case, $F(\psi, S)$ is equal to the R.H.S. of (7) with the summation carried out over all $j \neq i$. This is the case studied in [6].

4) $S=B_{0}\left(R_{1}\right)$ : This is our first random set example: the tagged transmitter only knows/uses the location of it nearest neighboring receiver, excluding $y_{0}$. We then have (8) with

$$
F(\psi, S)=\frac{1}{\frac{\left(R_{1} / r\right)^{\beta}}{T}+1-\psi}+C\left(\psi, \frac{R_{1}}{r}\right) .
$$

It is easy to see that $\psi\left(R_{1}\right)$ increases with $R_{1}$.

5) $S=B_{0}\left(R_{k}\right)$ : Here $k$ is a positive integer. The case $k=0$ boils down to $S=\emptyset$ and the case $k=\infty$ to $S=\mathbb{R}^{2}$. In terms of implementation, this setting is better than $S=B_{0}(R)$ since it allows one to tune the actual cardinality of the set of receivers taken into account in the MAP control. However there is no guarantee on the path-loss between the transmitter and the nodes in this set. In this case, we have (8) with

$$
F(\psi, S)=\sum_{p=1}^{k} \frac{1}{\frac{R_{p}^{\beta}}{r^{\beta} T}+1-\psi}+C\left(\psi, \frac{R_{k}}{r}\right) .
$$

6) $S=B_{0}\left(R_{k}\right) \cap B_{0}(R)$ : This two parameter stopping set enjoys the two practical properties mentioned above: (i) the number of receivers taken into account in the control of each transmitter is upper bounded by $k$; (ii) the mean path-loss to any receiver taken into account in the control is upper bounded by $R^{\beta}$. In this case, we have (8) with

$$
F(\psi, S)=\sum_{p=1}^{\min \left(k, B_{0}(R)\right)} \frac{1}{\frac{R_{p}^{\beta}}{r^{\beta} T}+1-\psi}+C\left(\psi, \frac{\min \left(R_{k}, R\right)}{r}\right) .
$$

If $\beta=4$ the R.H.S.s of (11)-(13) can be simplified using (10).

\section{Performance of the Optimal Control}

\section{A. MAP Distribution}

Using Theorem 3.1 and monotonicity arguments, it is not difficult to show that for all $0 \leq \rho \leq 1$

$$
\psi>\rho \quad \text { iff } \sum_{\substack{j \neq 0: \\ y_{j} \in S}} \frac{\rho}{b_{0 j}+1-\rho}+I(\rho, S)<1,
$$

where $I(\rho, S)=\lambda \int_{y \in \mathbb{R}^{2} \backslash S} \frac{\rho}{|y|^{\beta} / T r^{\beta}+1-\rho} \mathrm{d} y$. We now use this to derive the distribution of the optimal MAP. For all $0 \leq \rho \leq$ 
1 and stopping sets $S$, let

$$
L_{(\rho, S)}(x, y)=\frac{\rho}{\frac{\mid x-y^{\beta}}{\operatorname{Tr}^{\beta}}+1-\rho} \mathbf{1}(y \in S) .
$$

The indicator $\mathbf{1}\left(y_{i} \in S\right)$ can be thought as a mark associated with $y_{i} \in \bar{\Phi}$. However, these marks are not independent unless $S$ is a constant set, e.g., if $S=B_{0}\left(R_{1}\right)$. For all $0 \leq \rho \leq 1$ and stopping sets $S$, the shot noise field $J_{\bar{\Phi}}(\rho, S)$ associated with the response function $L_{(\rho, S)}(0, y)$ and the marked point process $\bar{\Phi}$ is $J_{\bar{\Phi}}(\rho, S)=\sum_{i} L_{(\rho, S)}\left(0, y_{i}\right)$. Notice that this shot noise is not that representing the interference at the origin. It rather measures the effect of the presence of a transmitter at 0 on the set of receivers in $S$. We have the following connection between the optimal MAP distribution and the shot noise $J_{\bar{\Phi}}$. For all $0<\rho<1$, $\mathbb{P}^{0}(\psi>\rho)=\mathbb{P}^{0}\left(J_{\bar{\Phi} \backslash\left\{y_{0}\right\}}(\rho, S)<1-I(\rho, S)\right)$, and $\mathbb{P}^{0}(\psi=$ $1)=\mathbb{P}^{0}\left(J_{\bar{\Phi} \backslash\left\{y_{0}\right\}}(1, S)<1-I(1, S)\right)$. From Slivnyak's theorem [3, Theorem 1.13], $\mathbb{P}^{0}\left(J_{\bar{\Phi} \backslash\left\{y_{0}\right\}}(\rho, S)<1-I(\rho, S)\right)=$ $\mathbb{P}\left(J_{\bar{\Phi}}(\rho, S)<1-I(\rho, S)\right)$, for all $0 \leq \rho \leq 1$. Thus, we get:

Theorem 4.1: For all $0<\rho<1, \mathbb{P}^{0}(\psi>$ $\rho)=\mathbb{P}\left(J_{\bar{\Phi}}(\rho, S)<1-I(\rho, S)\right)$ and $\mathbb{P}^{0}(\psi=1)=$ $\mathbb{P}\left(J_{\bar{\Phi}}(1, S)<1-I(1, S)\right)$.

\section{B. Examples}

1) Deterministic Sets: Since $\bar{\Phi}$ is a homogeneous Poisson point process, it follows from [3, Proposition 2.6] that the Laplace transform $\mathcal{L}_{J(\rho, S)}(s)$ of the shot noise $J_{\bar{\Phi}}(\rho, S)$ is:

$$
\mathcal{L}_{J(\rho, S)}(s)=\exp \left(-\lambda \int_{S}\left(1-\mathrm{e}^{-\frac{s \rho T r^{\beta}}{|y|^{\beta}+(1-\rho) T r^{\beta}}}\right) \mathrm{d} y\right) .
$$

For example, when $S=B_{0}(R)$ for a fixed $R$,

$$
\mathcal{L}_{J(\rho, S)}(s)=\exp \left(-\pi \lambda \int_{0}^{R^{2}}\left(1-\mathrm{e}^{-\frac{s \rho T r^{\beta}}{t^{\beta / 2}+(1-\rho) T r^{\beta}}}\right) \mathrm{d} t\right) .
$$

Moreover, if $\beta=4$, the Laplace transform $\mathcal{L}_{J(\rho, S)}(s)$ can be further simplified as

$$
\mathcal{L}_{J(\rho, S)}(s)=e^{-2 \pi \lambda \sqrt{(1-\rho) T} r^{2} \int_{v_{R}}^{1} \frac{1-\mathrm{e}^{-s \rho v^{2} /(1-\rho)}}{v^{2} \sqrt{1-v^{2}}} \mathrm{~d} v},
$$

where $v_{R}=\sqrt{(1-\rho) T} r^{2} / \sqrt{R^{4}+(1-\rho) T r^{4}}$.

Theorem 4.2: For all deterministic sets $S$, the optimal MAP of the typical node has for distribution

$$
\mathbb{P}^{0}(\psi>\rho)=\frac{1}{2 \pi} \int_{-\infty}^{\infty} \mathcal{L}_{J(\rho, S)}(i w) \frac{\mathrm{e}^{i w(1-I(\rho, S))}-1}{i w} \mathrm{~d} w,
$$

with $\mathcal{L}_{J(\rho, S)}(\cdot)$ given by (15). Similarly, $\mathbb{P}^{0}\{\psi=1\}$ is given by the R.H.S. with $\rho$ replaced by 1 .

Proof: Let $g_{\rho}(\cdot)$ denote the density of $J_{\Phi}(\rho, S)$. Then

$$
\mathbb{P}^{0}(\psi>\rho)=\int_{0}^{1-I(\rho, S)} g_{\rho}(t) \mathrm{d} t=\int_{-\infty}^{\infty} g_{\rho}(t) u(t) \mathrm{d} t,
$$

where $u(t)=1$ if $0 \leq t \leq 1-I(\rho, S)$ and 0 otherwise. Now from the Plancherel-Parseval theorem (see [3, Lemma 12.1])

$$
\mathbb{P}^{0}(\psi>\rho)=\frac{1}{2 \pi} \int_{-\infty}^{\infty} \mathcal{F}_{J(\rho, S)}(w) \mathcal{F}_{u}^{*}(w) \mathrm{d} w,
$$

with $\mathcal{F}_{A}(w)=\mathbb{E} \exp (-i w A)$ the Fourier transform of the real valued random variable $A$ and $B^{*}$ the complex conjugate of $B$.
The claim follows after substituting $\mathcal{F}_{u}(w)=\frac{1-\mathrm{e}^{-i w(1-I(\rho, S))}}{i w}$ and $\mathcal{F}_{J(\rho, S)}(w)=\mathcal{L}_{J(\rho, S)}(i w)$. Similarly, $\mathbb{P}^{0}(\psi=1)$.

2) $S=B_{0}\left(R_{1}\right)$ : Observe that $I\left(\rho, B_{0}(x)\right)=$ $2 \pi \lambda r^{2} \int_{x / r}^{\infty} \frac{\rho s}{\frac{s^{\beta}}{T}+1-\rho} \mathrm{d} s$ is increasing in $\rho$ and decreasing in $x$. For $0 \leq \rho \leq 1$, let

$\xi(\rho)=\inf \left\{x \geq 0: \frac{\rho}{x^{\beta} / \operatorname{Tr}^{\beta}+1-\rho}+I\left(\rho, B_{0}(x)\right)<1\right\}$.

Clearly, $\xi(\rho)$ is increasing in $\rho$. Using this and (14), we get that $\mathbb{P}^{0}(\psi>\rho)=\mathbb{P}^{0}\left(R_{1}>\xi(\rho)\right)$. From Slivnyak's Theorem, $\mathbb{P}^{0}\left(R_{1}>\xi(\rho)\right)=\mathbb{P}\left(R_{1}>\xi(\rho)\right)$. This together with the formula for the probability that the ball with radius $\xi(\rho)$ contains no point of a PPP of intensity $\lambda$ give:

Theorem 4.3: If $S=B_{0}\left(R_{1}\right)$, then $\mathbb{P}^{0}(\psi>\rho)=$ $\exp \left(-\lambda \pi \xi(\rho)^{2}\right)$ for $\rho<1$ and $\mathbb{P}^{0}(\psi=1)=\exp \left(-\lambda \pi \xi(1)^{2}\right)$. To illustrate the last theorem, assume that for a (small enough) $\rho, \xi(\rho)=0$. This means that all the nodes should attempt with probabilities lower bounded by $\rho$ in each slot. Similarly, assume that $\xi(1)=R^{*}$. Then all the nodes for whom the closest neighboring receivers are at distances greater than $R^{*}$ should transmit in every slot.

\section{Mean Utility}

We now provide an analytical expression for the mean optimized utility per unit space:

$$
\Theta^{S}=\lambda\left(\mathbb{E}^{0}\left[\log \left(\psi^{S}\right)\right]+\mathbb{E}^{0}\left[\log \left(q_{0}^{S}\right)\right]\right) .
$$

Let $f(\cdot)$ denote the distribution of $\psi^{S}$ (see Section IV-A). For all $t \in \mathbb{R}^{2}$, let $f_{t}$ be the distribution of $\psi^{S}$ with an extra receiver at $t$, i.e. the distribution of the solution of

$$
\frac{1}{\psi}=D(\psi, S)+\frac{\mathbf{1}(t \in S)}{1+\frac{|t|^{\beta}}{|r|^{\beta} T}-\psi}+\int_{y \in \mathbb{R}^{2} \backslash S} \frac{\lambda \mathrm{d} y}{1+|y|^{\beta} / T r^{\beta}-\psi}
$$

if it exists and $\psi^{S}=1$ otherwise. Notice that $f($.$) and f_{t}($. both depend on $S$. For each $S$ studied above, one gets an analytical expression for $f_{t}$ by the same technique as in $\S I V-A$.

Theorem 4.4: For all $S, \Theta^{S}$ is given by:

$$
\begin{aligned}
\Theta^{S} & =\lambda \int_{[0,1]} \log (u) f(\mathrm{~d} u) \\
& +\lambda \int_{t \in \mathbb{R}^{2}} \int_{u \in[0,1]} \log \left(1-\frac{u}{1+|t|^{\beta} / \operatorname{Tr}^{\beta}}\right) f_{t}(\mathrm{~d} u) \mathrm{d} t .
\end{aligned}
$$

Proof: We have

$$
\begin{aligned}
\mathbb{E}^{0}\left[\log \left(q_{0}^{S}\right)\right]=\mathbb{E}^{0}\left[\sum_{j \neq 0} \log \left(1-\frac{\psi^{S}}{1+b_{0 j}}\right)\right] \\
=\mathbb{E}^{0}\left[\sum_{j \neq 0} \log \left(1-\frac{\psi^{S}\left(\delta_{y_{j}}+\sum_{i \neq j, 0} \delta_{y_{i}}\right)}{1+\frac{\left|y_{j}\right|^{\beta}}{r^{\beta} T}}\right)\right] \\
=\lambda \int_{\mathbb{R}^{2}} \mathbb{E}^{0}\left[\log \left(1-\frac{\psi^{S}\left(\delta_{t}+\bar{\Phi}\right)}{1+\frac{\mid t \beta^{\beta}}{r^{\beta} T}}\right)\right] \mathrm{d} t
\end{aligned}
$$




$$
=\lambda \int_{\mathbb{R}^{2}} \int_{[0,1]} \log \left(1-\frac{u}{1+\frac{|t|^{\beta}}{r^{\beta} T}}\right) f_{t}(\mathrm{~d} u) \mathrm{d} t .
$$

In these equations, we first used (22) in Appendix to get (19), then stressed the fact that $\psi^{S}$ is a function of $\bar{\Phi}$ by writing $\psi^{S}=\psi^{S}\left(\sum_{i \neq 0} \delta_{y_{i}}\right)$ in (20), and finally used the fact that $\bar{\Phi}$ is Poisson and Campbell's formula to get (21).

\section{Convergence}

The aim of this section is to substantiate the claim that the control schemes of Section III can get arbitrarily close to the full information scheme. We illustrate this for the case of the deterministic stopping sets $B_{0}(R)$ by proving some continuity results of the main performance metrics when $R$ tends to infinity. When $R \rightarrow \infty$, we have

$$
\sum_{\substack{j \neq 0 \\ y_{j} \notin B_{0}(R)}} \frac{1}{\frac{\left(\left|y_{j}\right| / r\right)^{\beta}}{T}+1-\psi} \rightarrow 0, \quad \int_{R / r}^{\infty} \frac{s}{\frac{s^{\beta}}{T}+1-\psi} \mathrm{d} s \rightarrow 0 .
$$

It then follows from (9) and from standard (deterministic) calculus arguments which are skipped here that, $\mathbb{P}^{0}$ a.s., $\lim _{R \rightarrow \infty} \psi^{B_{0}(R)}=\psi^{\mathbb{R}^{2}}$, which proves the continuity of the MAP at infinity. The main result of this section is:

Lemma 4.1: $\Theta^{B_{0}(R)}$ tends to $\Theta^{\mathbb{R}^{2}}$ when $R \rightarrow \infty$.

Proof: In view of (17) and (19), this will hold if we can interchange the a.s. limits w.r.t. $R$ and the expectations in both terms of the R.H.S. of (17). In order to do so, thanks to the dominated convergence theorem, it is enough to show that each of the positive random variables $-\log \left(\psi^{B_{0}(R)}\right)$ and $-\log \left(q_{0}^{B_{0}(R)}\right)$ is uniformly bounded from above by some random variables with finite mean, where uniformity is w.r.t. $R$ large enough. For $-\log \left(\psi^{B_{0}(R)}\right)$, using the bound $-\log \left(\psi^{B_{0}(R)}\right) \leq 1 / \psi^{B_{0}(R)}$, we see that it is enough to prove the property for $1 / \psi^{B_{0}(R)}$. But we get from (9) and elementary monotonicity arguments that for all $R>1$, $\frac{1}{\psi^{B_{0}(R)}} \leq \frac{K_{1}}{1-\psi^{B_{0}(R)}}+A(R)$ with $K_{1}$ the number of receivers in $B_{0}(1)$ (excluding $y_{0}$ ) and

$$
A(R)=\sum_{j \neq 0: y_{j} \in B_{0}(R) \backslash B_{0}(1)} \frac{r^{\beta}}{T\left|y_{j}\right|^{\beta}}+2 \pi \lambda r^{2} \int_{R / r}^{\infty} \frac{s^{1-\beta}}{T} \mathrm{~d} s .
$$

This gives $\frac{1}{\psi^{B_{0}(R)}} \leq 1+K_{1}+A(R) \leq 1+K_{1}+A(1)$, which is the announced upper bound. The proof for $-\log \left(q_{0}^{B_{0}(R)}\right)$ is similar and is skipped.

\section{Numerical Evaluation and Simulation}

Simulation Setting: We consider a two dimensional square with side length $L$, and $N$ nodes placed independently over this square according to the uniform distribution; this corresponds to $\lambda=N / L^{2}$ in the stochastic geometry model ${ }^{2}$. Each node has its receiver randomly located on the unit circle around it, again as per the uniform distribution. Thus $r_{i i}=1$ for all $i$. We set $\alpha=4$ and $T=10$. To nullify the edge

\footnotetext{
${ }^{2}$ A finite snapshot of a Poisson random process would contain a Poisson distributed number of nodes. However, for large $\lambda L^{2}$, the Poisson random variable with mean $\lambda L^{2}$ is highly concentrated around its mean. Thus we can use $\lambda L^{2}$ nodes for all the realizations in our simulation.
}

effect, we take into account only the nodes falling in the $L / 2 \times L / 2$ square around the center while computing various metrics. While all other parameters remain, we vary $L$ and $N$ for different simulations. For each parameter set we calculate the average of the performance metric of interest over 1000 independent network realizations.

Joint Validation of the Analysis and the Simulation: For illustration, we consider $S=\mathbb{R}^{2}$ and study the c.d.f. $f_{t}$ of the MAP $\psi^{\mathbb{R}^{2}}$ (see Section IV-C). We set $L=40$ and $N=400$, which corresponds to $\lambda=0.25$. Figure 1 shows the plots for $\lambda=0.25$ and two values of $t: t=1$ and $t=10$. First, observe

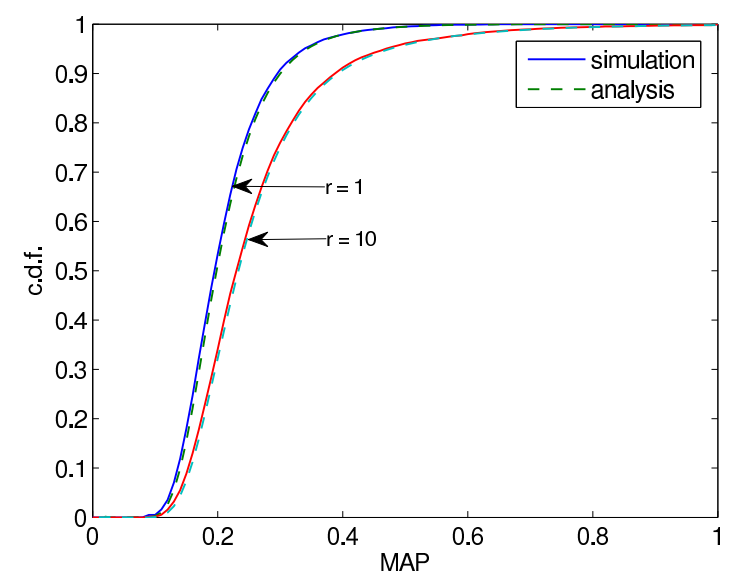

Fig. 1: C.d.f. $f_{t}$ of the MAP $\psi^{\mathbb{R}^{2}}$ with an extra receiver.

that the stochastic geometry based formula (see Theorem 4.2) matches simulation quite accurately. As expected, $f_{1}\left(\psi^{\mathbb{R}^{2}}\right) \geq$ $f_{10}\left(\psi^{\mathbb{R}^{2}}\right)$ for all $\psi^{\mathbb{R}^{2}}$, i.e., the tagged node (at origin) is more likely to be inactive for $t=1$.

Constant Stopping Sets: We illustrate here the variation of performance w.r.t. information on the case $S=B_{0}(R)$ through two sets of curves (both obtained by simulation). The top picture of Figure 2 quantifies the gains of the mean of the logarithm of the typical node's throughput as $R$ increases. In contrast, the bottom picture of Figure 2 plots the variations of the mean aggregate throughput per unit space (referred to as density of throughput) as a function of $\lambda$ when $R$ varies. A first observation, in line with [11], is that an increase of information does not necessarily lead to a better performance for this last metric (there is no reason for this to hold anyway as the schemes optimize the above logarithmic metric). This is however true for small $\lambda$, where the absolute gains are quite substantial.

Random Stopping Sets: In Figure 3, we plot the average aggregate throughput (sum of throughputs throughout the simulation window) under the optimal control $\pi^{S}$ for $S=\mathbb{R}^{2}, B_{0}\left(R_{1}\right)$ and $\emptyset$, and for $\lambda$ varying from 0.02 to 1 . This figure shows that knowing (and accounting for) the distance of the closest receiver only brings most of the potential gains in terms of aggregate throughput ${ }^{3}$. We also note that while the

\footnotetext{
${ }^{3}$ The scheme $S=B_{0}\left(R_{1}\right)$, or more precisely its variant $S=B_{0}\left(R_{1}\right) \cap$ $B_{0}(R)$, which is better in terms of implementation (see Section VI) hence provides a good candidate in this class.
} 

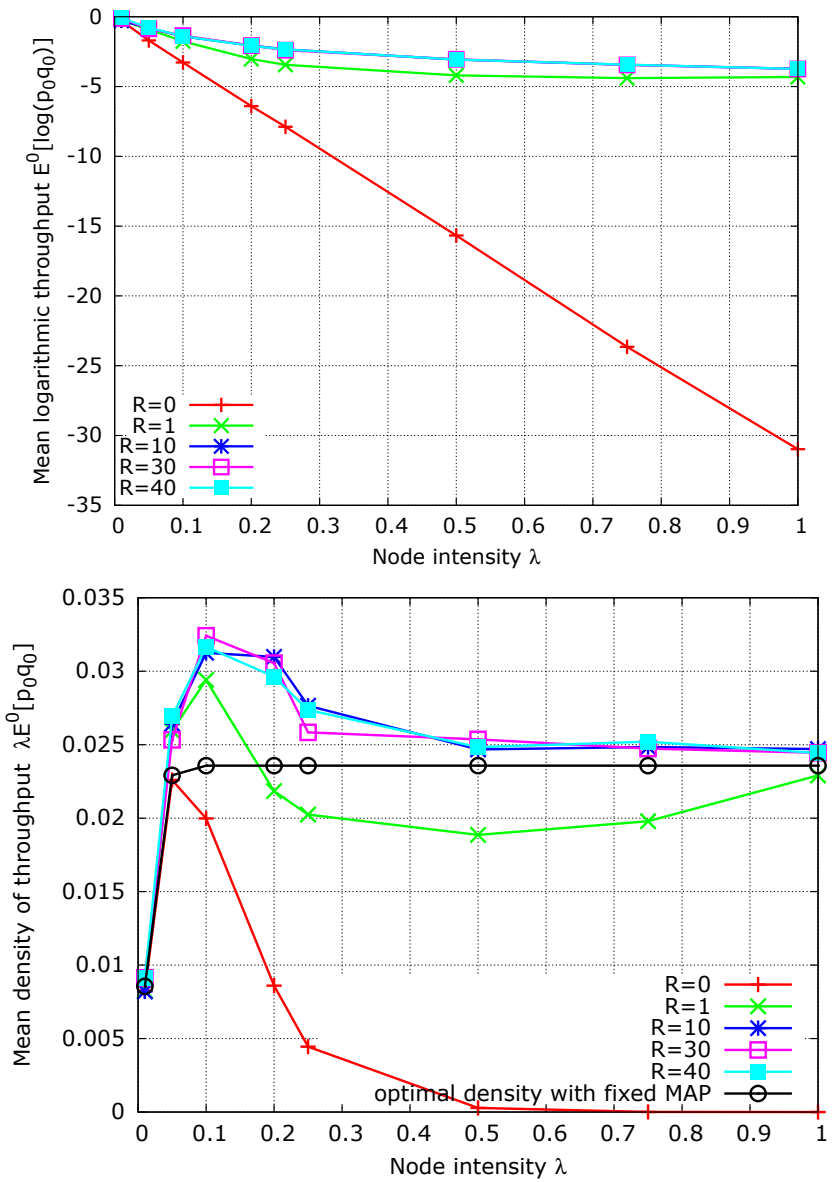

Fig. 2: Top: mean logarithmic throughput as a function of $\lambda$ for $S=B_{0}(R)$. Bottom: Density of throughput as a function of $\lambda$ for $S=B_{0}(R)$. The curve entitled "optimum density with fixed MAP" gives the result of the parametric optimization of the density of throughput performed in [4] for comparison.

gains are large for smaller node intensities, they diminish as $\lambda$ increases.

\section{IMPLEMENTATION}

The data required at transmitter 0 (the tagged transmitter) in order to solve the fixed point Equation (4) have two components: (1) the parameters $b_{0 j}$, for all $y_{j}$ in the stopping set $S$, which show up in the sum of the R.H.S., and (2) the intensity parameter $\lambda$ which shows up in the integral of this R.H.S. The estimation of $\lambda$ will not be discussed here. We will concentrate below on the mechanisms through which the sequence $b_{0 j}, y_{j} \in S$, on which the adaptation is based, can be estimated by the tagged transmitter. Assuming that $T$ and $r$ are known, the knowledge of $b_{0 j}$ is equivalent to that of the path-loss $\left|X_{0}-y_{j}\right|^{\beta}$ between the tagged transmitter and receiver $j$. We center the discussion on the case $S=B_{0}(R)$.

Our assumptions on the communication capabilities of the nodes are the usual ones for an Aloha MAC setting: (a) Each node (called transmitter or receiver until now) can actually both transmit and receive: transmitters transmit packets and receive acknowledgements, whereas receivers receive packets and transmit acknowledgements. (b) Each acknowledgement is transmitted with a known and constant power $P_{a}$. (c)

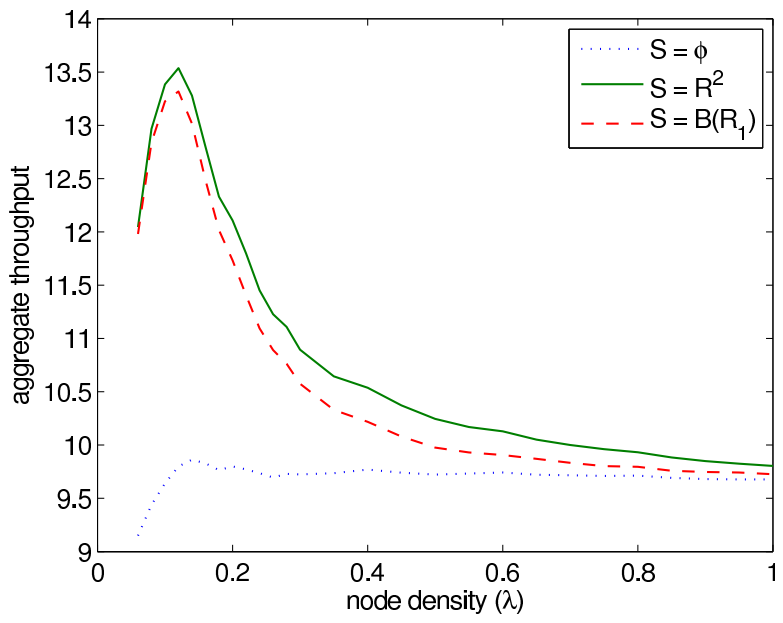

Fig. 3: Aggregate throughput as a function of intensity.

Each acknowledgement contains the identity of the transmitterreceiver pair. In addition, we assume here that the tagged receiver can decode all acknowledgements stemming from receivers at distance less than $R$. This assumption is debatable in general but justified for moderate $R .{ }^{4}$

The main idea discussed below consists in using the acknowledgements that are broadcast by all receivers to allow the tagged transmitter to estimate $\left|X_{0}-y_{j}\right|^{\beta}$ for all $y_{j} \in S$ (and in fact each transmitter to estimate in parallel his pathloss to each receiver in his own stopping set). For this, each transmitter decodes all acknowledgements and keeps a moving average of the powers at which acknowledgements stemming from node $j \in S$ were received. Since acknowledgements stemming from $j$ with $y_{j} \in S$ are assumed to be successfully decoded with probability 1 and since the identity of receiver $j$ is assumed to be contained in the acknowledgement, the tagged transmitter has the means to maintain this moving average. The $k$-th element in the time series of received powers is $P_{a} G_{j 0}^{(k)}\left|X_{0}-y_{j}\right|^{\beta}$, with the sequence $\left\{G_{j 0}^{(k)}\right\}_{k}$ made of i.i.d. exponential random variables of mean 1 (because of the Rayleigh fading assumption). By the strong law of large numbers, we have $\lim _{n \rightarrow \infty} \frac{1}{n} \sum_{k=1, n} P_{a} G_{j 0}^{(k)}\left|X_{0}-y_{j}\right|^{\beta}=$ $P_{a}\left|X_{0}-y_{j}\right|^{\beta} \quad$ a.s.. So the tagged node can indeed estimate $\left|X_{0}-y_{j}\right|^{\beta}$ from his moving average for all $y_{j}$ at a distance less than $R$, and hence $b_{0 j}$ for all $y_{j} \in S$.

\section{CONCLUSION}

This paper determines in an analytic way the performance of a Poisson network using a version of Aloha where each transmitter willingly adapts his medium access probability based on local information so as to reach a network wide proportional fairness. To the best of our knowledge, this is the first example of successful combination of stochastic geometry

${ }^{4}$ For instance, for $R$ of the order of $r$, since the data rate required for acknowledgements is much lower than that for payload packets, the SINR conditions for acknowledgements are easier to meet than those for packets, so this assumption is acceptable. 
and local adaptive protocol design aimed at optimizing a utility function within this Aloha setting. The particular case where the local information is limited to the interference incurred by the closest receiver is shown to be have some implementable variants and to bring most of the potential performance gain.

\section{APPENDIX}

We give here the proof of Theorem 3.1. From (1),

$$
\begin{gathered}
\mathbb{E}^{0}\left[\log \left(p_{0} q_{0}\right)\right]=\mathbb{E}^{0}\left[\log (\psi)+\sum_{X_{j} \neq 0} \log \left(1-\frac{p_{j}}{1+b_{j 0}}\right)\right] \\
=\mathbb{E}^{0}[\log (\psi)]+\mathbb{E}^{0}\left[\sum_{X_{j} \neq 0} \log \left(1-\frac{\psi}{1+b_{0 j}}\right)\right]
\end{gathered}
$$

where the second equality is due to the mass transport principle (cf. [2, Page 65]). Our goal is to maximize the last expression in function of $\psi$ under the restriction (2) for the given stopping set $S=S(\Phi, \bar{\Phi})$. For this, we split the sum in this expression into two terms depending on whether $y_{j} \in S$ or $y_{j} \notin S$. By the strong Markov property; cf [3, Definition 1.16]) and Campbell's formula for the Poisson point process $\bar{\Phi}$, we get that the second term of R.H.S. of (22) is equal to $\mathbb{E}^{0}\left[\int_{y \in \mathbb{R}^{2} \backslash S} \log \left(1-\frac{\psi}{1+|y|^{\beta} / \operatorname{Tr}^{\beta}}\right) \mathrm{d} y\right]$. We thus conclude that $\mathbf{P} \mathbf{F}^{S}$ is equivalent to the maximization of the following expectation in the considered class of functions $\psi(\cdot)$

$$
\begin{aligned}
\mathbb{E}^{0}[\log (\psi) & +\sum_{y_{j} \in S, j \neq 0} \log \left(1-\frac{\psi}{1+b_{0 j}}\right) \\
& \left.+\lambda \int_{y \in \mathbb{R}^{2} \backslash S} \log \left(1-\frac{\psi}{1+|y|^{\beta} / \operatorname{Tr}^{\beta}}\right) \mathrm{d} y\right] .
\end{aligned}
$$

It is obvious that maximizing the expression under the expectation for any given realization of $\bar{\Phi}$ (it does not depend on $\Phi)$ we will obtain a solution of $\mathbf{P} \mathbf{F}^{S}$. The derivative of this expression with respect to $\psi$ is equal to

$$
\frac{1}{\psi}-\sum_{y_{j} \in S, j \neq 0} \frac{1}{1+b_{0 j}-\psi}-\int_{y \in \mathbb{R}^{2} \backslash S} \frac{\lambda \mathrm{d} y}{1+|y|^{\beta} / T r^{\beta}-\psi} .
$$

It is continuous and decreasing in $\psi$ over $[0,1]$. This proves that the MAC policy $\psi^{S}$ is a solution of $\mathbf{P F}^{S}$. In order to prove that $\mathbb{E}^{0}\left[\psi^{S} q_{0}\right]>-\infty$ observe that the expectation (23) is finite $(>-\infty)$ for a constant MAC policy $\psi=$ const $\in(0,1)$.

Finally, in order to prove the uniqueness, suppose that for some other MAC policy $\psi^{\prime}(\cdot)$ the value of the expectation (23) is equal to that obtained for $\psi^{S}$; i.e., $\mathbb{E}^{0}\left[H\left(\psi^{S}(\bar{\Phi})\right)\right]=$ $\mathbb{E}^{0}\left[H\left(\psi^{\prime}(\bar{\Phi})\right)\right]$, where $H(\cdot)$ is the expression under the expectation in (23) as a function of the MAC policy. Then we have $\max \left(H\left(\psi^{S}(\cdot), H\left(\psi^{\prime}(\cdot)\right)-H\left(\psi^{\prime}(\cdot)\right) \geq 0\right.\right.$ and

$$
\begin{aligned}
& \mathbb{E}^{0}\left[\max \left(H\left(\psi^{S}(\bar{\Phi})\right), H\left(\psi^{\prime}(\bar{\Phi})\right)-H\left(\psi^{\prime}(\bar{\Phi})\right)\right]\right. \\
& =\mathbb{E}^{0}\left[\max \left(H\left(\psi^{S}(\bar{\Phi})\right), H\left(\psi^{\prime}(\bar{\Phi})\right)\right]-\mathbb{E}^{0}\left[H\left(\psi^{\prime}(\bar{\Phi})\right)\right]\right. \\
& \geq \max \left(\mathbb{E}^{0}\left[H\left(\psi^{S}(\bar{\Phi})\right)\right], \mathbb{E}^{0}\left[H\left(\psi^{\prime}(\bar{\Phi})\right)\right]\right)-\mathbb{E}^{0}\left[H\left(\psi^{\prime}(\bar{\Phi})\right)\right]=0
\end{aligned}
$$

since $\mathbb{E}^{0}\left[H\left(\psi^{\prime}(\bar{\Phi})\right)\right]=\mathbb{E}^{0}\left[H\left(\psi^{S}(\bar{\Phi})\right)\right]>-\infty$. Consequently $\max \left(H\left(\psi^{S}(\bar{\Phi})\right), H\left(\psi^{\prime}(\bar{\Phi})\right)=H\left(\psi^{\prime}(\bar{\Phi})\right)=H\left(\psi^{S}(\bar{\Phi})\right.\right.$ for almost all realizations of $\bar{\Phi}$. The fact that $H(\cdot)$ has one maximum in $[0,1]$ (attained for $\psi^{S}$ ) allows one to conclude that $\psi^{\prime}(\bar{\Phi})=\psi^{S}(\bar{\Phi})$ for almost all realizations of $\bar{\Phi}$.

\section{REFERENCES}

[1] N. Abramson. THE ALOHA SYSTEM: another alternative for computer communications. In Proceedings of the fall joint computer conference (AFIPS), Montvale, N.J, Nov. 1970.

[2] F. Baccelli and B. Blaszczyszyn. Stochastic Geometry and Wireless Networks Vol. II: Applications, volume 4 issue 1-2 of Foundations and Trends in Networking. NOW Publishers, 2009.

[3] F. Baccelli and B. Blaszczyszyn. Stochastic Geometry and Wireless Networks Volume I: Theory, volume 3 issue 3-4 of Foundations and Trends in Networking. NOW Publishers, 2009.

[4] F. Baccelli, B. Blaszczyszyn, and P. Muhlethaler. An Aloha protocol for multihop mobile wireless networks. IEEE Transactions on Information Theory, 52(2):421-436, February 2006.

[5] F. Baccelli, B. Blaszczyszyn, and P. Muhlethaler. Stochastic analysis of spatial and opportunistic Aloha. IEEE Journal on Selected Areas in Communications, 27(7):1105-1119, 2009.

[6] F. Baccelli and C. Singh. Adaptive spatial Aloha, fairness and stochastic geometry. In Proceedings of the Ninth Workshop on Spatial Stochastic Models for Wireless Networks, Spaswin 2013 - in conjunction with WIOpt'13, Tsukuba, Japan, May 2013.

[7] C. Bordenave, S. Foss, and V. Shneer. A random multiple access protocol with spatial interactions. In Proceedings of IEEE WiOpt, pages 1-6, Limassol, Cyprus, 2007.

[8] P. Gupta and A. Stolyar. Throughput region of randomaccess networks of general topology. IEEE Transactions on Information Theory, 58(5), 2012.

[9] B. Hajek. Stochastic approximation methods for decentralized control of multiaccess communications. IEEE Transactions on Information Theory, 31(2):176-184, March 1985.

[10] M. K. Hanawal, E. Altman, and F. Baccelli. Stochastic geometry based medium access games. In IEEE Infocom, Orlando, Florida, USA, March 2012.

[11] F.-T. Hsu and H.-J. Su. Channel-aware ALOHA with SINR capture: When is the knowledge of channel not helpful? In Proceedings of IEEE PIMRC, Toronto, Canada, Sept. 2011.

[12] N. Jindal, S. Weber, and J. Andrews. Fractional power control for decentralized wireless networks. IEEE Transactions on Wireless Communications, 7(22):5482-5492, 2008.

[13] F. Kelly, A. Maulloo, and D. Tan. Rate control in communication networks: shadow prices, proportional fairness and stability. Journal of the Operational Research Society, 49:237-252, 1998.

[14] A.-H. Mohsenian-Rad, V. W. Wong, and R. Schober. Optimal sinr-based random access. In Proceedings of IEEE Infocom, San Diego, CA, USA, March 2010.

[15] L. G. Roberts. ALOHA packet system with and without slots and capture. ACM SIGCOMM Computer Communication Review, 5(2):28-42, April 1975.

[16] X. Wang and K. Kar. Distributed algorithms for max-min fair rate allocation in aloha networks. In 42nd Annual Allerton Conference on Communication, Control, and Computing, Urbana, IL, USA, September-October 2004.

[17] X. Zhang and M. Haenggi. Random power control in poisson networks. IEEE Transactions on Communications, 60(9), 2012. 